Classification

Physics Abstracts

$87.20 \mathrm{Mj}-87.10+\mathrm{e}$

\title{
Nucleation Far from Equilibrium
}

\author{
Alain Pumir \\ Instıtut Non Linéaure de Nice, CNRS, 1361 route des Lucioles, 06560 Valbonne, France
}

(Recelved 23 May 1994, revised 6 May 1995, accepted 6 July 1995)

\begin{abstract}
Nucleation in phase transition is a classical problem. Close to the transition point, the critical radius is known to be much larger than the duffusion length In this paper, we focus on the case far from the critical point, as it is the case in a supercooled liquid, or in the related context of excitable media. We show that the physics of nucleation is different from the classical case. The critical radius is of the order of the diffusion length (in 2-dimensions), and smaller than the diffusion length (in 1-dimension) As a result, diffusion first leads to a decrease in amplitude of the initial seed, before a propagating pulse (or front) is initiated. This results in different laws for the critical radius The relevance of these effects to stimulation in biological tıssues, such as cardiac muscle, is discussed
\end{abstract}

\section{Introduction}

Front propagation is a common phenomenon in many physical systems. Examples include solidification of an undercooled melt, flame propagation (a particular case of excitable medium), and many hydrodynamic, pattern forming systems [1-3]. Because of its importance, the subject has received a great deal of theoretical attention, since the pioneering work of Kolmogorov, Petrovsky and Piskunov [4], and Fischer [5]. Particularly, much work has been devoted to the problem of velocity selection for a front describing how a system in an unstable state is invaded by a stable phase [6-8].

In a number of problems, especially in systems undergoing first order phase transitions, a front may propagate, leading to the invasion of the metastable phase by a more stable phase. In such systems, it is known that a small seed of radius $R$ of the more stable phase grows only if the radius $R$ is larger than a critical size, $R_{\mathrm{c}}$ [9]. Nucleation in first order phase transitions has been extensively studied [10-13]. Close to the transition point, the critical radius is known to be much larger than the width of propagating fronts (or equivalently, the diffusion length). In this case, the critical radius has been estimated by considering the motion of a curved front. The effect of curvature is to slow down the velocity of propagation. The critical radius can be simply estimated as the radius of curvature corresponding to zero velocity of propagation (see Ref. [12], see also Section 5.3 below). It has to be emphasized that this picture makes sense only when the radius of curvature is much larger than the diffusion length. 
In 1-dimension, this kind of approach leads to the conclusion that the critical radius is zero, which of course is not consistent with the assumptions that the critical radius is large compared to the diffusion length. Close to the point where the metastable state becomes unstable (i.e., far away from the phase transition point), the critical radius goes to zero. This leads to an interesting dynamics. Qualitatively, diffusion first prevails, therefore leading to a decrease of the amplitude of the initial perturbation. The amplifying effect of the nonlinearity becomes important only after this initial phase. On the other hand, close to a phase transition, the critical radius becomes large compared to the diffusion length (it diverges logarithmically with the distance to the phase transition point), so diffusion never dominates the dynamics.

The picture is qualitatively similar in 2-dimensions. One of our main results here is that far away from the transition point (close to the point where the metastable state becomes unstable), the critical radius is smaller than the width of the front, and as a result, the dynamics is qualitatively similar to what is observed in 1-dimension. In this regime, the classical predictions [10-13] generally overestimate the critical radius. As expected, the classical results (based on the curved front approximation) are recovered close to the phase transition point.

These results can be extended to the case of excitable media. The two problems (excitable media and bistable systems) can be treated within the same framework, using the well-known FitzHugh-Nagumo model [14] (two partial differential equatıons, first order in time) which is the simplest model describing impulse propagation in biological tissues. This system contains as a limit a model describing the dynamics of first order phase transitions (the Schlögl model [10]) with one partial differential equation, first order in tıme. The limit is obtained when the restoration time in the second equation (the refractory time) tends to infinity. This limit is known to be singular [13].

The notion of critical radius is no longer defined in excitable media, very close to the transition point. This is due to the fact that when the refractory time is finite, propagation becomes impossible close to the transition point [13]. Close to the point where the metastable state becomes unstable (which corresponds to a small excitation threshold, in the terminology of excitable media), the critical radius has a linear dependence on the excitation threshold (finite refractory time), but a square root dependence on the excitation threshold in the phase transition problem (infinite refractory time). In excitable media, when the excitation threshold is zero, the critical radius behaves like the power $-1 / 4$ of the refractory time.

Conceptually, nucleation is perhaps the simplest fully time-dependent problem. For this reason, a good understanding of its mechanisms may be useful to describe the complex, transient effects involved in a number of problems related to chemistry and biology. One of our main motivations here is cardiology. Various methods of stimulation of the tissue allow to treat a number of arrhythmias (defibrillation, Anti-Tachycardia Pacing, etc.) [15-17], and nucleation is an important issue there. In this context, we study the critical size of an excited domain, necessary to induce propagating waves.

Our results are organized as follows: in Section 2, we briefly introduce our model, recall some elementary considerations, and formulate the problem we are interested in. In Section 3 we show qualitatively what happens to a localized excited region larger than the critical width, when the critical width is small. Then, we present and discuss our results in 1 (Section 4), and 2-dimensions (Section 5). In Section 6 we briefly summarize and discuss our results.

\section{Formulation of the Problem}

The simplest model providing a qualitatively correct picture of propagation in excitable tissues is the FitzHugh-Nagumo model [14]. It involves two variables only: $e(x, t)$, the membrane potential, and $v(x, t)$, the inhibitory variable. They obey the following partial differential 
equation:

$$
\partial_{t} e=f(e)-v+D \nabla^{2} e
$$

and

$$
\partial_{t} v=\epsilon e
$$

where

$$
f(e)=A e(1-e)(e-u) .
$$

The diffusion coefficient, $D$, and the kinetic coefficient, $A$, essentially set the length and time scales. The small parameters, $\epsilon$, is the inverse time scale of the evolution of the variable $v$ (the so-called refractory time). Observe that when $\epsilon=0$, the system reduces to a model describing first order phase transition (the Schlögl model [10]). We restrict to the case where $0 \leq u \leq 1$. In this case, both $e=0$ and $e=1$ are stable equilibrium points, and $e=u$ is an unstable equilibrium point. The parameter $u$ is the excitation threshold. The inhibitor, $v$, does not diffuse in the case of action potential propagation. A very different limit, where the inhibitor diffuses faster than the propagator, describes a number of pattern formation problems in biology (see, e.g., Ref. $[18,19]$ ).

In a uniform state $(\nabla e \equiv 0)$, it is well known that equation (1) can be written as:

$$
\frac{\mathrm{d} e}{\mathrm{~d} t}=-\frac{\mathrm{d} \mathcal{F}(e)}{\mathrm{d} e},
$$

where $\mathcal{F}$ is a Lyapunov function ('free energy'). The positions of equilibrium correspond to the extrema of $\mathcal{F}$. When $u<1 / 2$ (respectively $u>1 / 2$ ), the minimum of $\mathcal{F}$ associated with the solution $e=0$ is lower (respectively higher) than the minimum of $\mathcal{F}$ at $e=1$. The point corresponding to the absolute minimum of $\mathcal{F}$ will be refered to here as the stable point, and the other relative minimum of $\mathcal{F}$ as the metastable point. In a system governed by equation (1), the stable phase tends to invade the metastable state. A remarkable solution describing propagation of a front, with a velocity $v=\sqrt{D A / 2}(1-2 u)$ in a system asymptotically at $x \rightarrow-\infty$ in the state $e=1$, and at $x \rightarrow+\infty$, in the state $e=0$, has been found in closed form (see, e.g., Ref. [20]). For $u=1 / 2$, the velocity of the front is zero. It is easy to see that in this case, the free energy, $\mathcal{F}$, is equal for $e=0$ and $e=1$, meaning that $u=1 / 2$ corresponds to the transition point. For this reason, we refer to the case when $u \rightarrow 1 / 2$ as close to a phase transition. In the following, we restrict ourselves to $u<1 / 2$, so $e=1$ is the stable state, and $e=0$ is the metastable state. Note that for excitable biological tissues, $u$ is small (of the order $01-0.2$ ), i.e., the system is close to the case where the metastable branch disappears, far from the transition point. This leads to qualitative differences, as we will demonstrate below.

When $\epsilon>0$, instead of fronts, the steadily propagating solutions of equations $(1,2)$ are pulses. The front solutions corresponding to $u$ close to $1 / 2$ disappear as soon as $\epsilon \neq 0$.

We consider here the problem of growth of an initial seed of the stable phase, $e=1$, in a system prepared in the metastable phase $e=0$. Elementary dimensional considerations show that the critical radius, $w_{\mathbf{c}}$, must be of the form:

$$
w_{c}=\sqrt{D / A} \phi_{d}(u, \epsilon)
$$

where $d=1,2$ is the space dimension.

The initial condition we used in this work is:

$$
e(x, t=0)=\exp -(x / w)^{2} .
$$

(and $v=0$ everywhere). The parameter $w$ is the width of the initial perturbation. With this choice of initial condition, it is possible to estimate the function $\phi_{d}(u, \epsilon)$, because a Gaussian 
a)

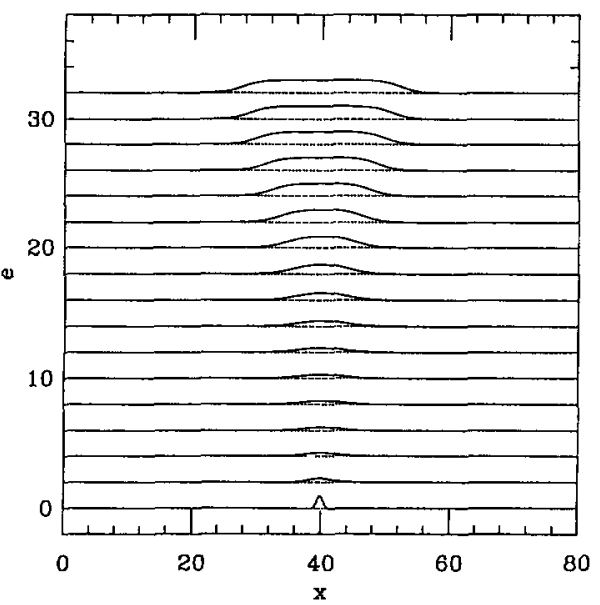

b)

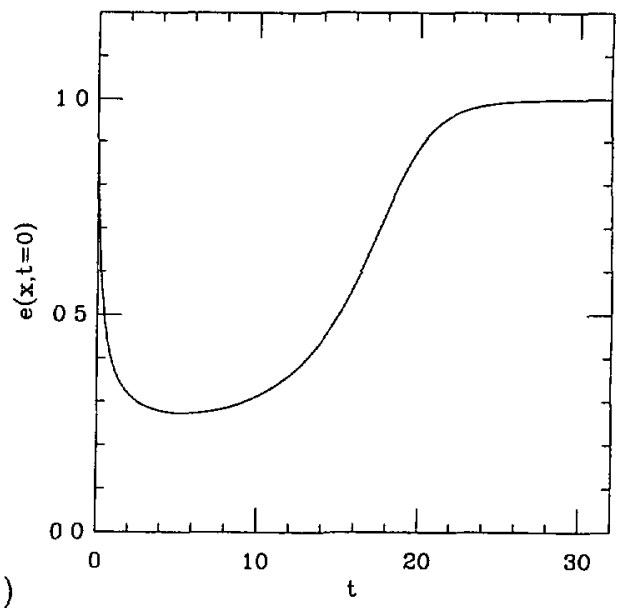

Fig. 1. - Evolution of a Gaussian initial condition (see Eq.(5)) In Figure 1a, solutions e(x) are shown at different times, separated by $\Delta t=2$. Figure $1 \mathrm{~b}$ shows the evolution of $e$ at the center of the system. The maximum of $e$ first decreases sharply, when the diffusive term dominates the nonlinear term. Finally, $e$ bounces up raises again, and the stable phase $(e=1)$ tends to invade the system. The dip in amplitude is due to the fact that the width is initially small. It disappears in the case where the critical radius is larger, $\imath e$, close to a phase transition point In this calculation, $u=01, D=1$ and $A=1$.

profile remains Gaussian under the diffusion equation. When $w$ is of the order of the diffusion length, diffusion first prevails. It is then reasonable to consider the reaction terms in equations $(1,2)$ as perturbations compared to the diffusion term $\left(\nabla^{2} e\right)$.

We have also investigated the problem numerically. Briefly, we describe our numerical methods. Equations $(1,2)$ were integrated numerically in 1-dimension, or in 2-dimensions, by imposing axisymmetry. The boundary conditions were either periodic (1-d), or of Neumann type. A standard Crank-Nicholson scheme, second order in time and space, was used. The code was checked by studying the decay of the initial condition (6), with the nonlinearity turned off, and by comparing the velocity of front propagation with the analytic result mentioned above. To determine the critical width, $w_{\mathrm{c}}=\phi_{1,2}(u, \epsilon)$, we integrated equations $(1,2)$, with equation (6) as the initial condition. For too small value of $w$, the initial condition shrinks to 0 , and for a large value of $w$, the small bump eventually evolves towards propagating pulse(s). The critical value, $w_{\mathrm{c}}$, is searched by dichotomy, once two values $w_{1}$ and $w_{2}$ are found, with $w_{1} \leq w_{\mathrm{c}} \leq w_{2}$ Extensive checks of our resolutions, in space and tıme, gave us confidence in our numerical results.

\section{Qualitative Aspects of the Evolution}

This short section illustrates our point, stated in the untroduction, that: i) the critical radius can be of size comparable to the width of the front (or even smaller in 1-dimension), and is) when the seed is larger than the critical radius, the amplitude of the solution may go down before it goes up again.

These qualitative features are illustrated by Figure 1. Figure 1a shows a series of curves $e(x, t)$, as a function of position $(x)$, at different times (n the 1-dimensional case). The values of the parameters are $u=0.1$ and $\epsilon=0$. Time increases upwards, and the time separation 
between two solutions is $\Delta t=2$. The initial pulse is visible in the lower record. In the next 7 records, the amplitude of the disturbance has significantly decreased. It increases later, after $\sim 8$ records. Figure $1 \mathrm{~b}$ shows the value of $e$, as a function of time, at $x=0$, the location of the initial bump (see Eq. (6)). The value of $e$ drops very quickly, and after a while, it increases again. As we will explain below in more detail, diffusion prevails when $w$ is small, and is responsible for the fast initial decay of the initial pulse. This behavior contrasts with the case where $w$ is sufficiently large, so the diffusion term is initially small, and essentially no drop of the maximum value of $e$ is observed.

It has to be emphasized that in 2-dimensions also, for $u \lesssim 0.2$, the evolution is qualitatively similar to what is seen in Figure 1. For this reason, the approximations used in references [11-13] are reasonable only in the regime where $u \gtrsim 0.3$. We now turn to a discussion of our results.

\section{1-Dimension Results}

4.1. Numerical Results. - We first discuss the results in 1-dimension. The function $\phi_{1}(u, \epsilon)$ is shown in Figure 2a for several values of $\epsilon$ : $\epsilon=0,0.01,0.02$ As expected, when $\epsilon$ increases, the critical radius increases. Also, propagation becomes impossible to close to $u=1 / 2$ when $\epsilon \neq 0[14]$, which explains why the upper curves, corresponding to $\epsilon=0.01,0.02$ do not extend to $u=1 / 2$.

When $u \rightarrow 0, \phi_{1}(u, \epsilon=0)$ goes to zero with an $1 / 2$ power (Fig. $2 \mathrm{~b}$ ). The function $\phi_{1}(u, \epsilon=0)$ diverges weakly when $u \rightarrow 1 / 2$, as $-\ln (1 / 2-u$ ) (Fig. 2c).

The function $\phi_{1}(u=0, \epsilon)$ behaves as $\epsilon^{1 / 4}$, as Figure $2 \mathrm{~d}$ demonstrates. Last, the behavior of $\phi_{1}(u, \epsilon)$ is shown near $u=0, \epsilon=0$. For $\epsilon_{0}>0$, the function $\phi_{1}\left(u, \epsilon_{0}\right)$ seems to have a linear behavior when $u \rightarrow 0$ (Fig. 2e).

The goal of the following three subsections is to give analytic arguments to explain our numerical observations.

4.2. The $\epsilon=0$, Small $u$ CASE. - Our numerical results show that when $u \rightarrow 0$, $\phi_{1}(u, \epsilon=0) \sim u^{1 / 2}$ (Fig. 2b). Understanding this result is the aim of this subsection.

The imporant remark is, again, that the critical width is small. The evolution of the initial condition is governed by a balance between diffusion $\left(\nabla^{2} e\right)$ and the reaction term, $f(e)$. Because the critical width, $w_{c}$, is small the diffusion term first prevails, and as a first approximation the solution simply obeys the diffusion equation (Fig. 1). It is well known that the diffusion equation transforms a Gaussian into a Gaussian: $e(x, t)=e_{0}(t) \exp \left(-\left(x / w_{0}(t)\right)^{2}\right)$, where $e_{0}(t)$ and $w_{0}(t)$ are the amplitude and width of the perturbation. Elementary algebraic manipulations lead, in the 1-dimensional case, to the following equations for the amplitude, $e_{0}(t)$ and for the width, $w_{0}(t)$ :

$$
\frac{\mathrm{d} e_{0}(t)}{\mathrm{d} t}=-2 \frac{e_{0}(t)}{w_{0}(t)^{2}}
$$

and

$$
\frac{\mathrm{d} w_{0}(t)}{\mathrm{d} t}=+\frac{2}{w_{0}(t)}
$$

It results from these two equations that $\mathrm{d}\left(w_{0}(t) e_{0}(t)\right) / \mathrm{d} t=0$ (which is a consequence of the conservation law), so it is convenient to rewrite equation (7) as:

$$
\frac{\mathrm{d} e_{0}(t)}{\mathrm{d} t}=-2 \frac{e_{0}(t)^{3}}{w^{2}}
$$




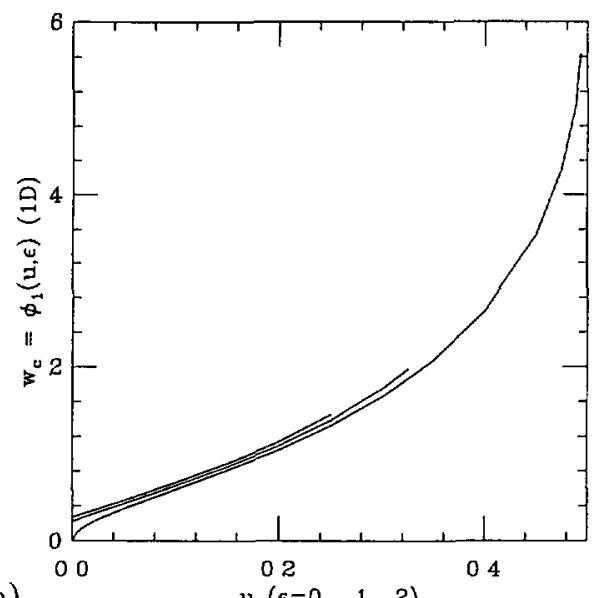

a)

u $(\epsilon=0,1,2)$

b)
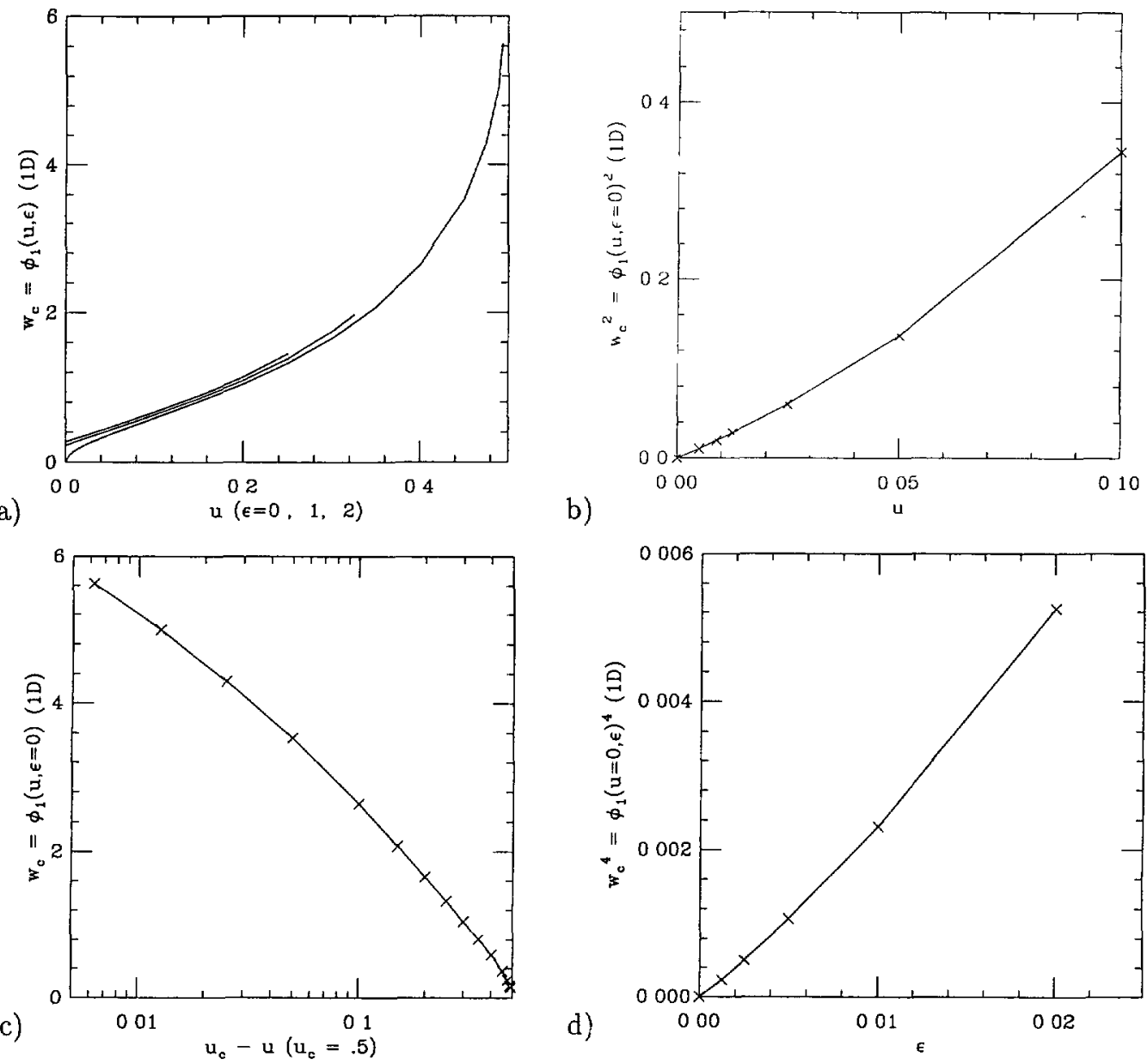

d)
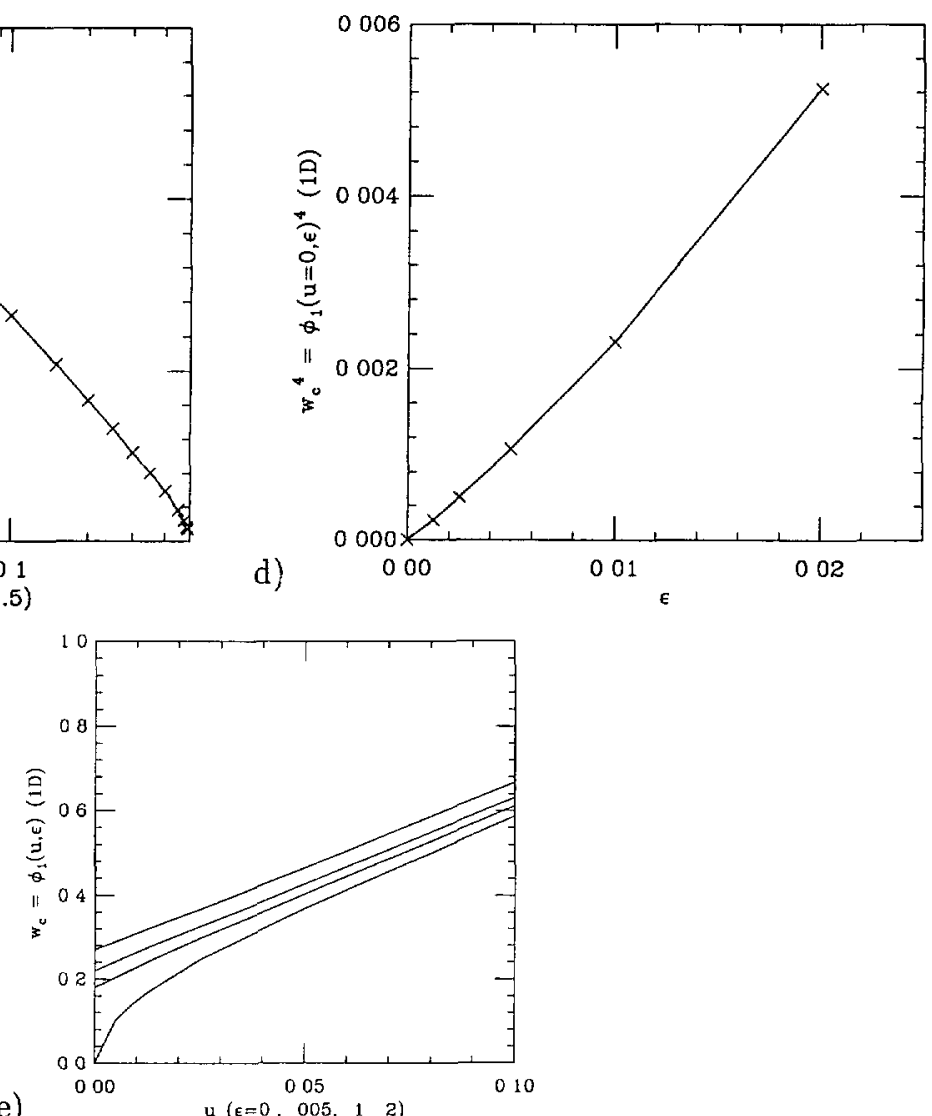

Fig 2. - Figure 2a shows the dependence of the critical width of a pulse, $\phi_{1}(u, \epsilon)$, in 1-dimension, as a function of the parameter $u$ for $\epsilon=0$ (lower curve), $\epsilon=001$ and $\epsilon=0.02$ (upper curve) Figure 2b shows the dependence of $\phi_{1}(u, \epsilon=0)$ as a function of $\sqrt{u}$ close to $u=0$. Figure 2c shows $\phi_{1}(u, \epsilon=0)$ as a function of $-\ln (1 / 2-u)$ Figure $2 \mathrm{~d}$ shows the $\epsilon^{1 / 4}$ dependence of $\phi_{1}(u=0, \epsilon)$. Last, Figure $2 \mathrm{e}$ shows a blow-up of the region close to $u=0$ and $\epsilon=0$. When $\epsilon \neq 0$, the function $\phi_{1}(u, \epsilon)$ behaves approximately linearly with $u$ for small values of $u$ 
where the initial condition, $e_{0}(0)=1$ and $w_{0}(0)=w$ have been taken into account (see Eq. $(6)$ ). Equation (9) describes the evolution of the amplitude of the Gaussian. We now estimate the effect of the reaction term, as in equation (3). Assuming that the effect of reaction term is weak, so the shape of the bump is not too much affected, one can write the following approximate equation for the amplitude of the solution:

$$
\frac{\mathrm{d} e_{0}(t)}{\mathrm{d} t}=-2 \frac{e_{0}(t)^{3}}{w^{2}}+e_{0}(t)\left(e_{0}(t)-1\right)\left(u-e_{0}(t)\right)
$$

It is a simple matter to study the behavior of the solution of this Ordinary Differential Equation (ODE), as a function of the parameter $w$. The polynomial on the Right Hand Side (RHS) of equation (10) always has a root, at $e_{0}=0$. When $w=\infty$, there are two other roots, at $e_{0}=u$ and $e_{0}=1$. The largest root is stable, for the evolution described by equation (10). When $w$ decreases, the two roots move towards $e_{0}=0$, and get closer to one another. They merge at $e=2 u /(1+u)$, for $w=\bar{w}$, with

$$
\bar{w}=\frac{\sqrt{8 u}}{(1-u)} .
$$

When $w<\bar{w}$, the RHS of equation (10) is negative for $0 \leq e_{0} \leq 1$, so the solution of equation (10) tends to zero. When $w>\vec{w}$, the right hand side of equation (10) is negative between $+\infty$ and the largest root of the RHS of equation (10), and positive between the two non zero roots. As a consequence, the solution of equation (8) cannot reach 0 . This simple picture suggests that for $w \leq \bar{w}$, the initial perturbation will eventually decay, whereas for $w \geq \bar{w}$, the solution could grow again. This suggests that $w_{\mathrm{c}} \sim \bar{w}$. Obviously the simple approximation that lead to equation (10) cannot always hold. Because of the nonlinearities, the shape of the bump cannot remain Gaussian forever. However, we could check that the picture just described has some validity, by comparing the time derivative of the maximum value of $e$, minus the reaction term, $f(e)$, to the RHS of equation (9). The agreement was found better and better when $u \rightarrow 0$, at least for short times. Eventually, the solution differs from its approximated form, therefore rendering the argument no better than qualitative.

It is easy to modify our analysis, so as to treat the case where the initial amplitude is arbitrary. In this case, $w$ should be replaced by the product $\left(e_{0}(0) w\right)$ in equations $(9,10)$. At fixed radius, $w$, this suggests that there is a threshold in amplitude of excitation, given by $e_{0}(0) w=\Phi_{1}$.

Again, we emphasize that the picture presented here is significantly different from the picture usually put forward to treat the nucleation problem.

4.3. The $\epsilon=0, u \rightarrow 1 / 2$ CASE. - The limit where the system is close to the a first order transition is considered next $(u \rightarrow 1 / 2)$ We present an analytic argument to explain the logarithmic dependence of $\phi_{1}(u, \epsilon=0)$ in this case (Fig. 2c).

To proceed, it is useful to remember the existence of an unstable, steady solution, obtained by solving the ODE:

$$
\partial_{x}^{2} e(x)+e(x)(e(x)-1)(u-e(x))=0
$$

When $u \rightarrow 1 / 2$, the unstable solution has a maximum: $2 / 3(1+u-\sqrt{(2-u)(1 / 2-u)})$, which is of order 1 . The length of the domain where $e>1 / 2$ diverges logarithmically when $u \rightarrow 1 / 2$. This solution is unstable, in the sense that when it is dilated a bit, the stable phase will tend to completely invade the system, whereas when it is compressed a bit, the system will return to the solution uniformly equal to 0 . To obtain a solution developing into a pair of traveling fronts, one needs to initiate a pulse larger than the steady, unstable solution. Since the spatial extension of this solution grows like $-\ln (1 / 2-u)$, when $u \rightarrow 1 / 2$, the initial width must be 


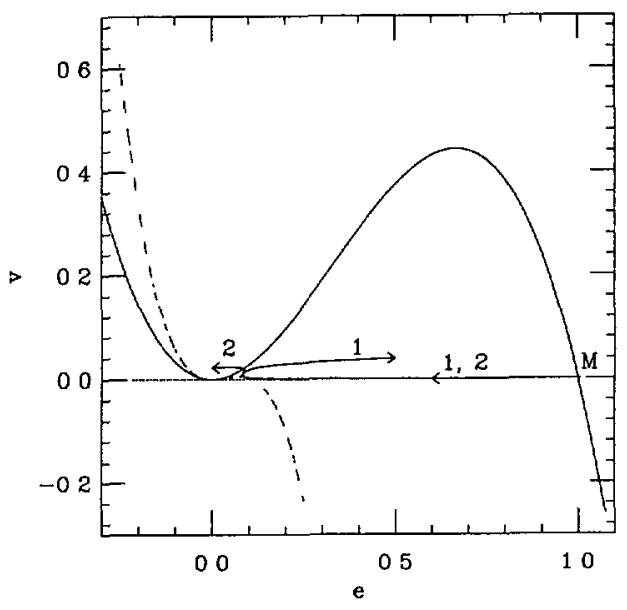

Fig. 3. - The phase plane, $(e, v)$ The fast nullclne, $v=f(e)$ is shown for $u=0$. Two solutions, one with initial size greater than the critical radius (1), and one with initial size smaller than the intial size (2) can be seen. The two solutions start at the point $M=(1,0)$, and move first almost parallel to the $v=0$ axis, until the reach the curve $v=g(e)=-e^{2}(e-1)-e^{3} / w^{2}$, shown in dashed lines. The two solutions are very close untll they meet the curve $v=g(e)$, and differ completely afterwards.

larger than a quantity, growing logarithmically to be able to initiate propagation. Very close to $u=1 / 2$, and for $w \approx w_{\mathrm{c}}$, it happened that our numerical solutions fell so close to the steady solution that the solution seemed to have converged to the (very weakly) unstable solution.

4.4. THE $\epsilon \neq 0$ CASE . - Last, we explain the features observed when $\epsilon \neq 0$, namely: i) $\phi_{1}(u=0, \epsilon) \sim \epsilon^{1 / 4}$ when $\epsilon \rightarrow 0$ (Fig. 2d), and ii) $\phi_{1}\left(u, \epsilon=\epsilon_{0}\right)$ behaves linearly with $u$ for small $u$, when $\epsilon_{0} \neq 0$ (Fig. $2 \mathrm{e}$ ).

It is possible to treat the case $\epsilon \neq 0$ by using some elaboration of the ideas presented in the case $\epsilon=0$. For small values of $\epsilon$ and $u$ (hence for small values of $w_{\mathrm{c}}$ ), we again approximate the solution by a Gaussian, with a width $w_{0}(t)$ and an amplitude $e_{0}(t)$. In the same spirit, $v_{0}(t)$ denotes the amplitude of the inhibitory variable at the maximum $(x=0)$. Equation (10) becomes:

$$
\begin{gathered}
\frac{\mathrm{d} e_{0}}{\mathrm{~d} t}(t)=-2 \frac{e_{0}(t)^{3}}{w^{2}}+e_{0}(t)\left(e_{0}(t)-1\right)\left(u-e_{0}(t)\right)-v_{0}(t) \\
\frac{\mathrm{d} v_{0}}{\mathrm{~d} t}(t)=\epsilon e_{0}(t)
\end{gathered}
$$

To describe the evolution of this system of ordinary differential equations, it is convenient to introduce the phase space $\left(e_{0}, v_{0}\right)$ (Fig. 3 ).

When $\epsilon=0$, the point representing the solution of equations $(13,14)$ moves along the $v_{0}=0$ line. When $\epsilon$ is small, but non zero $(0<\epsilon<<1)$, the point $\left(e_{0}, v_{0}\right)$ moves up slowly at first above the $v_{0}=0$ line. If the trajectory $\left(e_{0}(t), v_{0}(t)\right)$ is always above the curve $v_{0}=$ $e_{0}\left(e_{0}-1\right)\left(u-e_{0}\right)-2 e_{0}^{3} / w^{2}$, then, the amplitude $e_{0}$ will relax to zero, implying that no propagation is initiated. If, on the other hand, the trajectory $\left(e_{0}(t), v_{0}(t)\right)$ intersects the curve $v_{0}=e_{0}\left(e_{0}-1\right)\left(u-e_{0}\right)-2 e_{0}^{3} / w^{2}$, then, the point $e_{0}$ will bounce up, and propagation will be initiated. To study the critical width, it is natural to focus on the region where the function $g\left(e_{0}\right) \equiv e_{0}\left(e_{0}-1\right)\left(u-e_{0}\right)-2 e_{0}^{3} / w^{2}$ is maximum. Our strategy is then to introduce scaled variables in this region, and to derive an inner problem for the scaled variables. 
To this end, we first consider the case $u=0$. The maximum of the function $g\left(e_{0}\right)$ is located at $e_{\max }=2 / 3\left(w^{2} /\left(2+w^{2}\right)\right)$. We introduce the change of variables:

$$
e_{0} \equiv \frac{2}{3} \frac{w^{2}}{\left(2+w^{2}\right)}(1+\delta e)
$$

so the function $g\left(e_{0}\right)$ becomes:

$$
g\left(e_{0}\right)=\frac{4}{27} \frac{w^{4}}{\left(2+w^{2}\right)^{2}}\left(1-3 \delta e^{2}-\delta e^{3}\right) .
$$

Introducing the ratio $A \equiv \epsilon\left(2+w^{2}\right)^{2} / w^{4}$, the scaled inhibitory variable $\delta v$ by: $v_{0} \equiv\left(w^{4} /(2+\right.$ $\left.\left.w^{2}\right)^{2}\right) \delta v$, and the scaled time $\bar{t} \equiv\left(w^{2} /\left(2+w^{2}\right)\right) t$, equations $(13,14)$ become:

$$
\partial_{\bar{t}} \delta e=\frac{2}{9}\left(1-3 \delta e^{2}-\delta e^{3}\right)+\delta v
$$

and:

$$
\partial_{\bar{t}} \delta v=\frac{2}{3} A(1+\delta e)
$$

The inner problem, equations $(17,18)$, depends on the parameter $A$ only. This suggests that the critical width is given by a condition of the form:

$$
\epsilon \frac{\left(2+w^{2}\right)^{2}}{w^{4}}=A_{c}
$$

where $A_{\mathrm{c}}$ is given by the solution of equations (17,18). Equation (19) reduces, for small $\epsilon$, to $w \sim \epsilon^{1 / 4}$, as found numerically (Fig. 2d). Our analysis suggests that the slow time scale, when $\epsilon \rightarrow 0$, is $t \sim \epsilon^{-1 / 2}$, and in the inner region, $e \sim \epsilon^{1 / 2}$ and $v \sim \epsilon$. These predictions were explicitely checked by superposing $\epsilon^{-1 / 2} e$ and $\epsilon^{-1} v$ as a function of $\epsilon^{1 / 2} t$, for $w$ slightly above $(5 \%)$ the critical radius. The various curves superpose well, therefore confirming the results of our analysis. No attempt was made to solve explicitely equations $(17,18)$, since it provides at best a qualitative description of the evolution.

Using the same scaling as before, when $u \neq 0$, it is easy to see that provided $u<<\epsilon^{1 / 2}$, the extra term in equation (17) is of order $\left(u / \epsilon^{1 / 2}\right)(1+\delta e)$. When $u / \epsilon^{1 / 2} \rightarrow 0$, this term is a small, regular perturbation which explains why, when $\epsilon \neq 0, \phi_{1}(u, \epsilon)$ behaves linearly as a function of $u$, for small $u$.

We again emphasize that for $\epsilon \neq 0$, propagating solutions do not exist for $u$ close to $1 / 2$. This means that the problem of nucleation does not make sense in this case, although "pulses" could be observed transiently sometime in our numerics.

\section{2-Dimension Results}

5.1. Numerical Results. - We now turn to the results in 2-dimensions. The function $\phi_{2}(u, \epsilon)$ is shown in Figure $4 \mathrm{a}$ for several values of $\epsilon: \epsilon=0,0.01$ and 0.02 . As it was the case in 1-dimension, the critical radius increases with $\epsilon$. Propagation becomes impossible for $\epsilon \neq 0$, when $u \rightarrow 1 / 2$. As a consequence, the curves corresponding to $\epsilon=0.01$ and $\epsilon=0.02$ do not extend to $u=1 / 2$. When $u \rightarrow 0, \phi_{2}(u, \epsilon=0) \sim \alpha+\beta \sqrt{u}$, as Figure $4 \mathrm{~b}$ demonstrates. Figure $4 \mathrm{c}$ shows $1 / \phi_{2}(u, \epsilon=0)$ as a function of $u$. It strongly suggests that $\phi_{2}(u, \epsilon=0) \sim 1 /(1 / 2-u)$ when $u \rightarrow 1 / 2$.

The function $\phi_{2}(u=0, \epsilon)$ goes to $\alpha$ like $\epsilon^{1 / 4}$, as shown in Figure $4 d$. Last, as it was the case in 1-dimension, for $\epsilon_{0}>0$, the function $\phi_{2}\left(u, \epsilon_{0}\right)$ behaves linearly with $u$, for small values of $u$.

In the next three subsections we give analytic arguments to explain our numerical observations. 


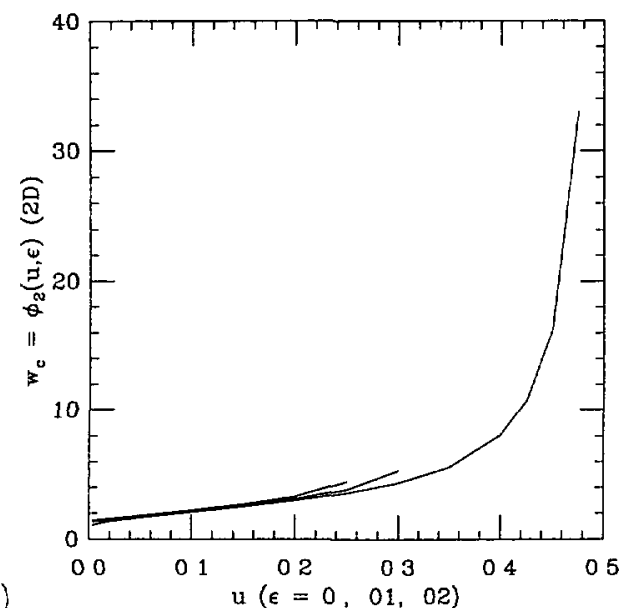

a)
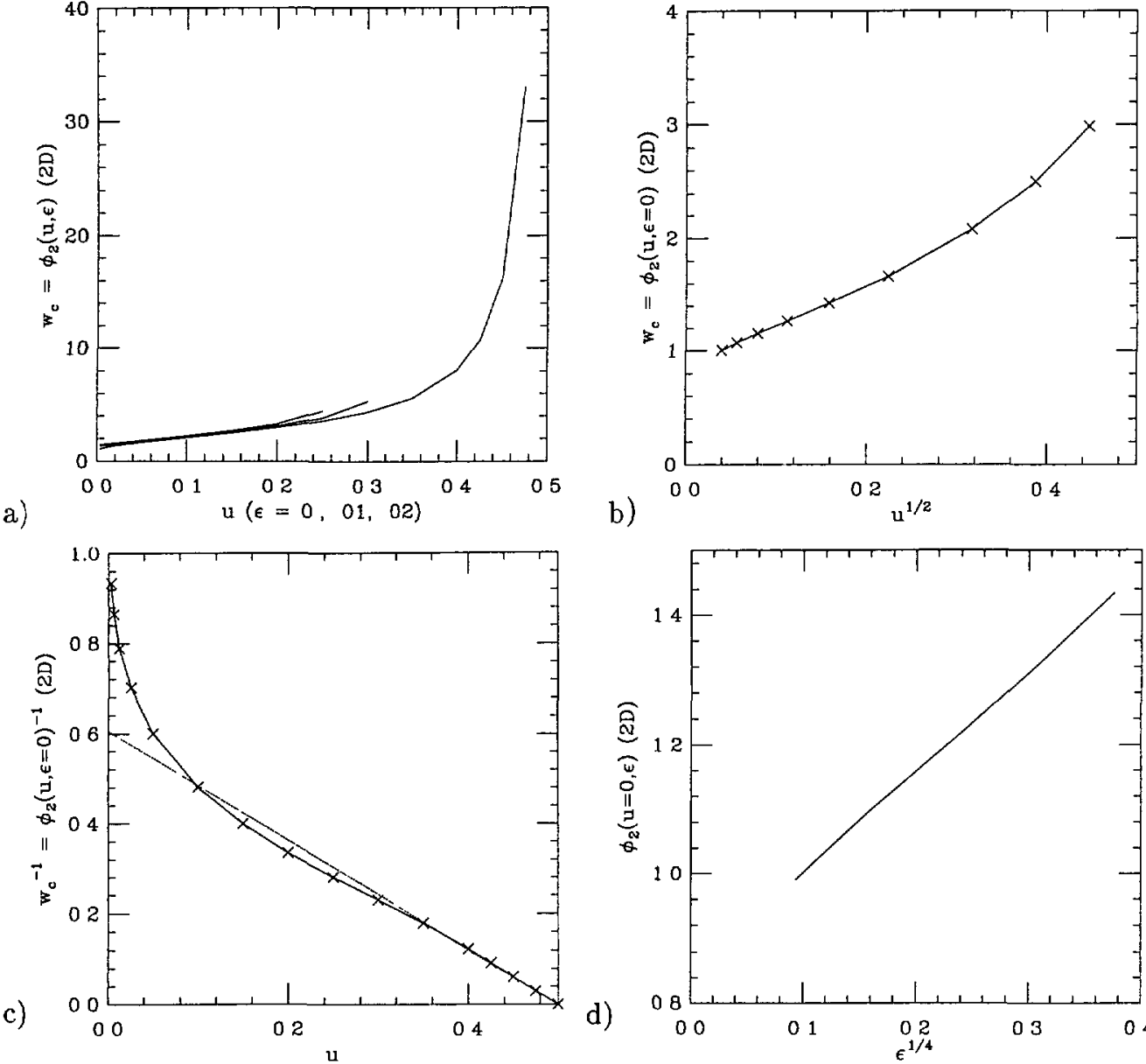

d)
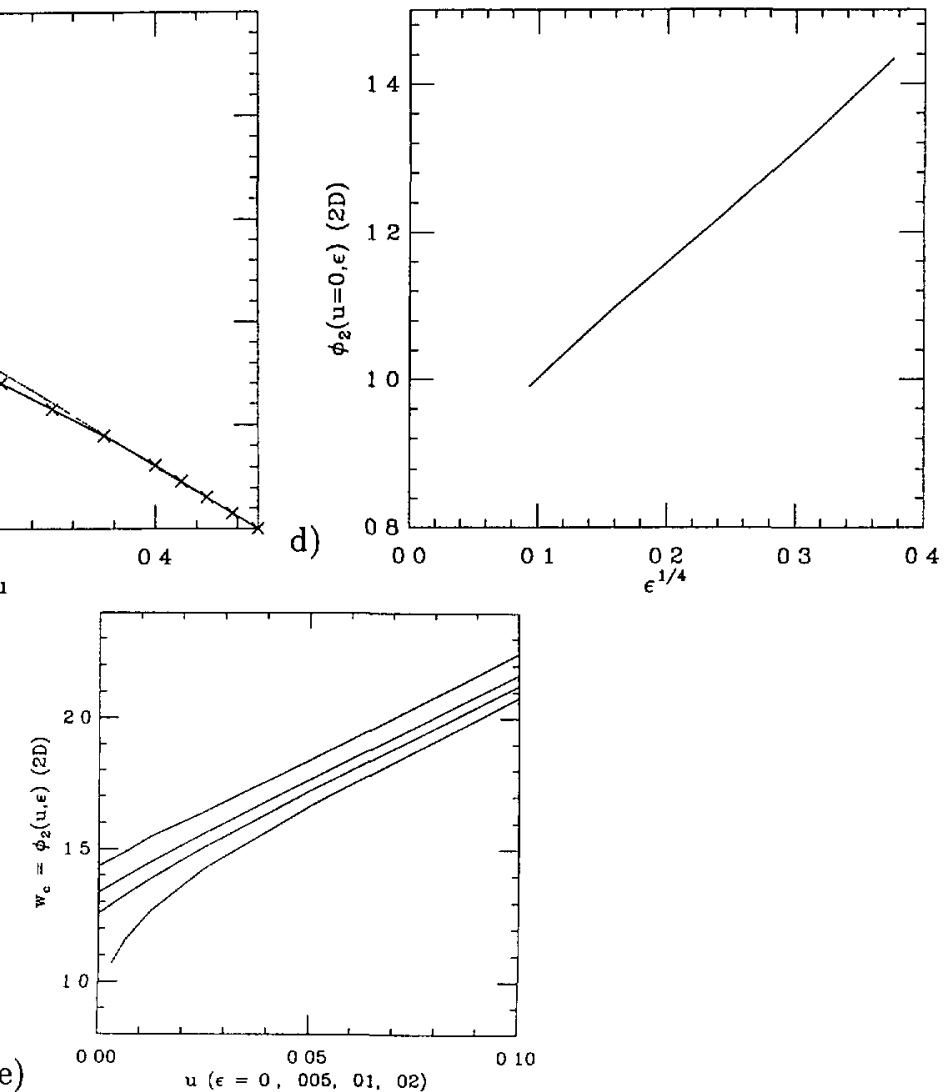

Fig 4. - Figure 4a shows the dependence of the critical width of a pulse, $\phi_{2}(u, \epsilon)$, in 2-dimensions, as a function of the parameter $u$ for $\epsilon=0$ (lower curve), $\epsilon=001$ and $\epsilon=0.02$ (upper curve) Figure $4 \mathrm{~b}$ shows the dependence of $\phi_{2}(u, \epsilon=0)$ as a function of $\sqrt{u}$ close to $u=0$, and Figure $4 \mathrm{c}$ shows $1 / \phi_{2}(u, \epsilon=0)$ as a function of $(u-1 / 2)$ In Figure 4c, the dashed line corresponds to the asymptotic behavior $\phi_{2}(u) \sim 0.82522 /(1 / 2-u)$, which is very close to the value predicted by using equation (26), with the correction described in the text $(1 / \sqrt{2 \ln 2}=0.84932)$. Figure $4 \mathrm{~d}$ shows the $\epsilon^{1 / 4}$ dependence of $\phi_{2}(u=0, \epsilon)$ close to $\epsilon=0$. Finally, Figure 4e shows the region close to $u=0$ and $\epsilon=0$ When $\epsilon \neq 0$, the function $\phi_{2}(u, \epsilon)$ behaves approximately linearly with $u$ for small values of $u$ 
5.2. The $\epsilon=0$, Small $u$ CASE. - Again, we consider first the case $\epsilon=0$. Figure $4 \mathrm{~b}$ shows that $\phi_{2}(u, \epsilon=0) \approx \alpha+\beta u^{1 / 2}$. In this subsection, we present an approximate argument to explain this dependence.

Our numerical results show that the critical size, $\phi_{2}(u, \epsilon=0)$ seems to go to a finite limit when $u \rightarrow 0$. It is therefore not a priori obvious that the approximations used to understand the limit $u \rightarrow 0$ in 1-dimension are applicable. However, the qualitative aspect of the evolution shown in Figure 1 was also found in 2-dimension, at least for small values of $u(u \lesssim 0.2)$. It is therefore interesting to modify the analysis of the 1-dimensional problem, since, as we show now, it provides some insight on the small $u$ limit.

Again, we first consider the case $\epsilon=0$. For a narrow bump (small $w$ in Eq.(6)), the diffusion term in equation (1) first dominates, and the reaction term can be treated, at least for a while, as a perturbation. As it is the case in 1-dimension, an (axisymmetric) Gaussian initial condition remains Gaussian: $e(r, t)=e_{2 \mathrm{D}}(t) \exp \left(-\left(r / w_{2 \mathrm{D}}(t)\right)^{2}\right)$. A straightforward substitution in the diffusion equation leads to the ordinary differential equation for $e_{2 \mathrm{D}}(t)$ and $w_{2 \mathrm{D}}(t)$ :

$$
\frac{\mathrm{d} e_{2 \mathrm{D}}(t)}{\mathrm{d} t}=-4 \frac{e_{2 \mathrm{D}}(t)}{w_{2 \mathrm{D}}(t)^{2}}
$$

and

$$
\frac{\mathrm{d} w_{2 \mathrm{D}}(t)}{\mathrm{d} t}=+\frac{2}{w_{2 \mathrm{D}}(t)}
$$

These two equations imply that $\mathrm{d}\left(w_{2 \mathrm{D}}^{2}(t) e_{2 \mathrm{D}}(t)\right) / \mathrm{d} t=0$, so equation (20) can be rewritten as:

$$
\frac{\mathrm{d} e_{2 D}(t)}{\mathrm{d} t}=-4 \frac{e_{2 \mathrm{D}}(t)^{2}}{w^{2}}
$$

where the initial condition $e_{2 D}(t=0)=1$ and $w_{2 D}(0)=w$ have been taken into account. The approximate equation for the evolution of the amplitude of the bump, obtained by adding up the effect of diffusion (Eq.(22)), and the advection term, $f(e)$ reads:

$$
\frac{\mathrm{d} e_{2 \mathrm{D}}(t)}{\mathrm{d} t}=-4 \frac{e_{2 \mathrm{D}}(t)^{2}}{w^{2}}+e_{2 \mathrm{D}}(t)\left(e_{2 \mathrm{D}}(t)-1\right)\left(u-e_{2 \mathrm{D}}(t)\right)
$$

The term originating from the diffusion equation is quadratic in 2-dimensions, instead of cubic in 1-dimension. The qualitative behavior of this simple ODE can be easily determined by studying the roots of its RHS. For large $w$, the RHS of equation (23) has three roots, including one at 0 , which is always stable and two roots greater than 0 . When $w$ decreases, the two positive roots get close to one another, and merge for $\bar{w}_{2 \mathrm{D}}=2 /(1+u-2 \sqrt{u})^{1 / 2}$ (the corresponding root is $e_{2 \mathrm{D}}=\sqrt{u}$ ). For $w \leq \bar{w}_{2 \mathrm{D}}$, the solution of the equation decreases to 0 , whereas for $w \geq \bar{w}_{2 D}$, the solution of equation (23) decreases until it reaches the largest root of the RHS of equation (23). Of course, the validity of the approximations which have lead us to equation (23) eventually become questionable. Even so, the results of this simple analysis predicts the right kind of dependence of $\phi_{2}(u, \epsilon=0)$ close to $u=0$

5.3. The $\epsilon=0, u \rightarrow 1 / 2$ CASE. - As we have explained before, when the system is close to the transition point $(u \approx 1 / 2)$, the critical nucleus is large, and the approximations used in references [11-13] are a proro valid. Indeed, as shown below, our results do agree with the predictions of these references.

The limit of $\phi_{2}(u, \epsilon=0)$ in the other case $(u \rightarrow 1 / 2)$ can be understood from the following simple considerations. In 2-dimensions, and for an axisymmetric solution, equation (1) reads:

$$
\partial_{t} e(r, t)-\frac{1}{r} \partial_{r} e(r, t)=\partial_{r}^{2} e(r, t)+e(r, t)(e(r, t)-1)(u-e(r, t)) .
$$


The modification, compared to the 1-dimensional case, comes from the advection term, with velocity $1 / r$, in the Left Hand Side (LHS) of equation (24). When the front is located at a distance $r$, much larger than the width of the front, the term $1 / r$ is roughly constant. As a consequence, the effective velocity of propagation is equal to the velocity of the front in 1-dimension, minus $1 / r$. Therefore, a seed of radius $R$ will be able to grow only if $1 / R<c=$ $\sqrt{1 / 2}(1-2 u)$. This shows that the order of magnitude of the critical radius must scale as $1 /(1 / 2-u)$, as found numerically.

Note that this phenomenological approach rests entirely on the fact the the normal component of the velocity of a front, $v_{\mathrm{n}}$, and the radius of curvature, $r_{\mathrm{c}}$, are related via:

$$
v_{\mathrm{n}}=V-D / r_{\mathrm{c}}
$$

where $V$ is the velocity of propagation in a 1-dimensional medium [13]. As such, it can also be applied to an excitable medium.

A straight application of equation (25) for determining the critical nucleus would lead to:

$$
r_{\mathrm{c}}=\frac{1}{\sqrt{2}(1 / 2-u)} .
$$

The coefficient of the inverse power law in equation (26) is significantly different from the coefficient we find numerically The discrepancy can be explained by the fact that, for $u \sim 1 / 2$, only the parts of the initial condition larger than $1 / 2$ are amplified by the reaction term. This suggests that $r_{c}$, in equation (26), should be compared with $w_{c} \times \sqrt{\ln 2}$. Once this correction is taken into account, the computed coefficient of the inverse power law differ from the approximated one by less than $3 \%$. Significant discrepancies exist between $\phi_{2}(u)$ and the asymptotic behavior, $\sim 1 /(1 / 2-u)$, away from $u$ close to $1 / 2$. They imply that predictions based on equation (25) (see, e.g. Ref. [13]) generally overestimate the critical radius.

5.4. The $\epsilon \neq 0$ CASE. - To treat the $\epsilon \neq 0$ case, one may, as in 1-dimension, extend our analysis to incorporate the effect of the inhibitor variable, $v_{2 \mathrm{D}}(t)$. Equation (23) then becomes:

$$
\begin{gathered}
\frac{\mathrm{d} e_{2 \mathrm{D}}(t)}{\mathrm{d} t}=-4 \frac{e_{2 \mathrm{D}}(t)^{2}}{w^{2}}+e_{2 \mathrm{D}}(t)\left(e_{2 \mathrm{D}}(t)-1\right)\left(u-e_{2 \mathrm{D}}(t)\right)-v_{2 \mathrm{D}}(t), \\
\frac{\mathrm{d} v_{2 \mathrm{D}}(t)}{\mathrm{d} t}=\epsilon e_{2 \mathrm{D}}(t) .
\end{gathered}
$$

One may again work close to the maximum of the polynomial in the right hand side of equation (27), and derive an inner problem, similar to what had been done in 1-dimension. The analysis, completely similar to what had been done in 1-dimension, see equations (15-19), leads to the conclusion that the critical width, $w_{\mathrm{c}}$, scales as $w_{\mathrm{c}} \sim \epsilon^{1 / 4}$, in agreement with the numerics. Also, the time scale of the problem diverges like $\epsilon^{-1 / 2}$, a prediction which agrees with the numerical results. However, the scaling of $e$ and $v$ are not correctly predicted by our analysis. The numerical solutions of equations $(1,2)$ suggest that $e \sim \epsilon^{1 / 2}$ and $v \sim \epsilon^{1 / 2}$, contrary to our simplified model, which instead suggests that $e \sim \epsilon^{1 / 4}$ and $v \sim \epsilon^{3 / 4}$. This shows the limit of our analysis, and calls for a more elaborate theoretical treatment.

The question of stability of an axisymmetric, growing nucleus remains to be addressed.

\section{Conclusion}

We have investigated nucleation in bistable and in excitable media. Our results allow us to identify two different regimes. Close to a first order phase transition point, the critical radius 
is large, compared to the diffusion length. In this regime, and in dimension $d \geq 2$, the classical results give an accurate prediction of the critical radius (the 1-dimensional case requires a special treatment). On the other hand, away from the critical point, close to the point where the metastable state disappears, or equivalently, for small excitation threshold, the critical radius is of the order of, or smaller than the width of the front. This leads to a dynamics qualitatively different from the case where the system is close to a phase transition, and to quantitatively different predictions for the critical radius, both in the phase transition case and in the excitable media case. When $\epsilon$ (the inverse refractory time) is non zero, the critical radius increases sharply when the excitation threshold is zero (like $\epsilon^{1 / 4}$ ). Because propagation is not possible when $\epsilon>0$, close to the phase transition point, the critical radius is not defined there

Although in excitable media (e.g. for a nerve fiber), the excitation threshold is typically small we wish to stress here that the other limit (close to a phase transition) is also relevant for fundamental problems in biology. The inhibitory variable is typically non zero, so the system is effectively above the resting point in phase plane $(e, v)$, corresponding to an increase of the excitation threshold. It is a common situation for pattern formation in biology (morphogenesis), hormone regulation of biochemical processes, interaction of excitation and inhibition in neurons in the brain, propagation excitation in striated, smooth and cardiac muscle [21].

\section{Acknowledgments}

My interest for this problem originated in discussions with V. Krinsky. It is a pleasure to thank him for his help and encouragements. I also benefited from discussions with P. Clavin and J.M. Flesselles.

\section{References}

[1] Pelcé P, Dynamics of curved fronts (Academic Press, 1988)

[2] Cross M. and Hohenberg P.C., Rev. Mod. Phys. 65 (1993) 851.

[3] Clavin P., Annu. Rev. Fluid Mech. 26 (1994) 321.

[4] Kolmogorov A, Petrovsky I. and Piskunov N, Bull. Unıv. Moscow, Ser. Int. Sec. A 1 (1937) 1

[5] Fischer R, Ann. Eugentcs 7 (1937) 355

[6] Aronson D.G. and Weinberger H.F., Adv. Math 30 (1978) 33.

[7] Ben-Jacob E., Brand H., Dee G., Kramer L. and Langer J S., Physica D 14 (1985) 348.

[8] van Saarloos W, Phys. Rev. A 37 (1988) 211 and Phys. Rev. A 39 (1989) 6367

[9] Landau L D and Lifshitz E M., Statistical Physics, $3^{\text {rd }}$ Edition (Pergamon Press, Oxford, 1980).

[10] Schlögl F, Z. Physık 253 (1972) 147

[11] Nitzan A , Ortoleva P. and Ross J., Faraday Symp. Chem. Soc. 9 (1974) 241.

[12] Malchow H. and Schımansky-Geier L., Noise and Diffusion in bistable nonequilibrium systems (Teubner-Verlag, Berlin, 1985).

[13] Mikhailov A S , Foundation of synergetics I Distributed active systems (Springer, Berlin, 1990).

[14] FitzHugh R., Broph J 1 (1961) 445

[15] Starmer C F., Biktashev V N, Romashko D.N., Stepanov M.R., Makarov O N. and Krınsky V I, Bıophys. J. 65 (1993) 1775.

[16] Krinsky V.I., Plaza F. and Voigner V., to be published in Phys. Rev (1995). 
[17] Winfree A.T., Biomathematics, Springer, Vol. 100, S Levin, Ed. (1995).

[18] Koch A J and Meinhart H., Rev. Mod Phys. 66 (1994) 1481.

[19] Kerner B.S. and Osipov V.V., Sov. Phys. Usp. 32 (1989) 101 and 33 (1990) 679

[20] Murray J.D., Mathematical Biology (Springer, New York, 1989).

[21] Alberts B , Bray D., Lew1s J., Raff M., Roberts K. and Watson J.D., Molecular biology of the cell (Garland Publishig Inc., New York, 1989). 